\title{
BALANCE SEDIMENTARIO DEL EMBALSE DE RIBARROJA
}

\author{
ÁLVARO TENA ${ }^{1 *}$, DAMIÀ VERICAT ${ }^{1,2}$, RAMÓN J. BATALLA ${ }^{1,3,4}$ \\ ${ }^{1}$ Fluvial Dynamics Research Group (RIUS), Universidad de Lleida (UdL), Lleida, España. \\ ${ }^{2}$ Centre de Ciencia i Tecnologia Forestal de Catalunya (CTFC), Solsona, España. \\ ${ }^{3}$ Institut Català de Recerca de l'Aigua (ICRA), Girona, España. \\ ${ }^{4}$ Facultad de Ciencias Forestales y Recursos Naturales, Universidad Austral de Chile, Valdivia, Chile.
}

RESUMEN: El objetivo de este estudio ha sido construir un balance de sedimentos del embalse de Ribarroja, situado en el tramo inferior del río Ebro. Para ello, se han analizado datos continuos de caudal y transporte de sedimentos en suspensión tomados en los principales ríos que desembocan en el embalse, así como en la salida de la presa, para el período 2008-2011. La carga de sedimentos estimada aguas arriba del embalse de Mequinenza fue de $0.66 \times 10^{6} \mathrm{ta}^{-1}$, mientras que la salida estimada fue de $0.04 \times 10^{6} \mathrm{t} \mathrm{a}^{-1}$. El aporte de sedimentos proveniente de los grandes afluentes pirenaicos, aunque altamente regulados, Segre y Cinca fue de $0,16 \times 10^{6} \mathrm{t} \mathrm{a}^{-1}$, mientras que los ríos intermitentes que fluyen naturalmente desde la Cordillera Ibérica (Matarraña y Algars) suministraron una carga media de $760 \mathrm{t} \mathrm{a}^{-1}$. La carga de sedimentos en suspensión atrapada en los embalses de Mequinenza y Ribarroja para el período de estudio se estimó en $0,78 \times 10^{6} \mathrm{t} \mathrm{a}^{-1}$, lo que implica una reducción de ca. $95 \%$ de la carga de sedimentos del río. Los resultados ilustran el papel de los embalses en la interrupción de la transferencia de sedimentos en el tramo bajo del Ebro, en un contexto de déficit sedimentario estructural, tras los cambios generalizados de uso del suelo (reforestación) que se produjeron en la cuenca a partir de los años cuarenta del siglo pasado.

\section{Sediment balance of the Ribarroja Reservoir}

\begin{abstract}
The aim of this study was to build up a sediment budget of the Ribarroja Reservoir, which is located in the lower reach of the River Ebro. For this purpose, we analysed continuous discharge and suspended sediment transport data at several river sections entering the reservoir and at the dam outlet, for the period 2008-2011. The sediment load estimated upstream of the Mequinenza reservoir was $0.66 \times 10^{6} \mathrm{t} \mathrm{a}^{-1}$, whereas the estimated output was $0.04 \times 10^{6} \mathrm{t} \mathrm{a}^{-1}$. The sediment input coming from the large but highly regulated Pyrenean tributaries (Segre and Cinca) was $0.16 \times 10^{6} \mathrm{t} \mathrm{a}^{-1}$, whereas intermittent rivers naturally flowing from the Iberian Range (Matarraña and Algars) supplied an average load of $760 \mathrm{t} \mathrm{a}^{-1}$. The suspended sediment load trapped in the Mequinenza and Ribarroja reservoirs for the study period was estimated at $0.78 \times 10^{6} \mathrm{ta}^{-1}$, which implies a reduction of ca. $95 \%$ of the sediment load of the river. The results illustrate the role of reservoirs in interrupting the sediment transfer in the lower reach of the Ebro, within a context of structural sediment deficit, following widespread land use changes (afforestation) that took place in the catchment after the 1940s.
\end{abstract}

Palabras clave: Balance sedimentario, embalses, río Ebro, sedimento en suspensión.

Key words: Sediment balance, reservoirs, Ebro River, suspended sediment.

Recibido: 29 septiembre 2020

Aceptado: 7 enero 2021 
*Correspondencia: Álvaro Tena. Fluvial Dynamics Research Group (RIUS), Universidad de Lleida (UdL), 25198, Lleida, España. E-mail address: alvaro.tena @udl.cat

\section{Introducción}

El impacto antrópico en sistemas fluviales se ha convertido en un tema central para gestores y científicos en las últimas décadas, favoreciendo el incremento de estudios para minimizar sus efectos o restaurar impactos. Entre los impactos destacan los que las grandes presas ejercen sobre la dinámica hidro-sedimentaria en ríos de todo el mundo. En este contexto, la retención de sedimentos en los embalses, y el corte en la transferencia de sedimentos hacia aguas abajo, se ha identificado como uno de los impactos más notables en el ecosistema fluvial, convirtiéndose en uno de los principales responsables de la tendencia decreciente de la transferencia de sedimentos entre las masas continentales y los océanos (e.g. Milliman y Syvitski, 1992; Meybeck y Ragu, 1997; Walling y Fang, 2003; Walling 2006, Milliman y Farnsworth, 2011). Vorosmarty et al. (2003) indicaron que, a nivel global, más de la mitad $(53 \%)$ de la carga sedimentaria transportada por los ríos queda actualmente retenida en los embalses i.e. $73 \mathrm{~km}^{3} \mathrm{a}^{-1}$ (Syvitsky y Kettner, 2011). En el caso del Mississippi se estima que casi 20 millones de toneladas de sedimentos se sedimentan y quedan retenidas anualmente en sus embalses, o del río Yangtze, en el que tan solo una presa, la de las Tres Gargantas, atrapa 34 millones de toneladas de sedimentos por año, lo que equivale al $31 \%$ de la carga sedimentaria transportada por el río (Hu et al., 2009). Milliman y Farnsworth (2011) describen diversos ejemplos (Colorado, Indus, Río Grande, Nilo) sobre el efecto de los grandes embalses en la carga sedimentaria de los ríos. En el caso del río Colorado, la presa Glen Canyon provocó una disminución de aproximadamente el 99,5\% en la cantidad de sedimento fino que circulaba por el Gran Cañón (Topping et al., 2000).

La región mediterránea presenta tasas de producción de sedimento elevadas a escala global (Dedkov y Mozzherin, 1992), alcanzando en algunos casos $>3000 \mathrm{t} \mathrm{km}^{-2} \mathrm{a}^{-1}$ por ej., el río Ombrone, Italia (Milliman y Farnsworth, 2011). Los usos del agua y la variabilidad hidro-climática de la región han sido el motivo histórico de la construcción de embalses para la regulación fluvial, principalmente a lo largo del siglo XX. Los usos de los embalses son múltiples, aunque el almacenaje de agua para riego ha sido el principal, sobre todo en España. Poulos y Collins (2002) ya observaron una reducción en la aportación sedimentaria a la cuenca del Mediterráneo de casi un 50\% desde mediados del siglo XX, asociado, entre otras razones (por ejemplo, cambios en los usos del suelo), al atrapamiento de una parte importante de la carga sólida de los grandes ríos, como el Po, el Ródano, el Ebro y el Nilo por parte de las grandes presas. Milliman y Farnsworth (2011) aumentan la reducción hasta el 75\%.

En este estudio se destaca que el río Ebro ha experimentado una importante reducción en su carga sedimentaria i.e. 95\%, especialmente en su tramo bajo, tras el cierre del complejo de presas Mequinenza-Ribarroja-Flix a final de los años 1960 (para más detalles, ver Batalla et al. 2004 sobre los efectos hidrológicos de las presas, y Batalla y Vericat (2011) sobre los cambios en el flujo de sedimentos en el conjunto de la cuenca sedimentaria debido, entre varias causas, a la retención en embalses). Existen numerosos estudios recientes sobre el transporte de sedimentos y los procesos geomorfológicos en el bajo Ebro en relación con el complejo de presas (e.g. Vericat y Batalla, 2005; Vericat y Batalla, 2006; Vericat et al., 2006; Rovira e Ibañez, 2007; Tena et al., 2011; Tena et al., 2013; Tena y Batalla, 2013; Rovira et al., 2015). A diferencia de los trabajos ya existentes, esta investigación se centra en el análisis de las entradas y salidas de sedimento fino del embalse de Ribarroja, incluyendo la cuantificación de las cargas sedimentarias del río Ebro, así como de los principales afluentes al embalse. Este balance ha sido elaborado principalmente a partir de series en continuo de sedimento en suspensión obtenidas entre 2008 y 2011 a la salida de cada una de las principales subcuencas que desembocan en el embalse de Ribarroja, y aguas abajo de la presa. El estudio se complementa con datos obtenidos anteriormente aguas arriba del embalse de Mequinenza. El objetivo es construir un balance sedimentario del embalse que permita evaluar sus efectos sobre la transferencia de sedimentos desde la cuenca hacia aguas abajo. Los 
resultados tienen interés para determinar la situación sedimentaria reciente del embalse y para re-evaluar las acciones prácticas de restauración ambiental que se pueden llevar a cabo en el tramo bajo del río Ebro.

\section{2. Área de estudio}

El Ebro es uno de los grandes ríos que desembocan en el Mar Mediterráneo (Fig. 1A), sólo superado en longitud por el Nilo. La cuenca del Ebro es, además, la más grande de la península Ibérica, con una superficie de $85.534 \mathrm{~km}^{2}$. El río fluye con dirección NW-SE a lo largo de $910 \mathrm{~km}$, desde la Cordillera Cantábrica hasta el delta del Ebro a través del cual desemboca en el Mar Mediterráneo. La altitud de la cuenca varía desde $>3400$ m s.n.m. en el Pirineo central hasta el nivel del mar en el delta (Fig. 1B). La precipitación media anual es de $600 \mathrm{~mm}$, aunque la variabilidad espacial y temporal es muy alta, variando desde $>2000 \mathrm{~mm} \mathrm{año}^{-1}$ en el Pirineo central hasta $<300 \mathrm{~mm}$ año ${ }^{-1}$ en el centro de la Depresión. El régimen hidrológico del Ebro es pluvio-nival, con máximos en invierno y primavera, mientras que los mínimos se dan en verano (Tena y Batalla, 2013). El caudal medio anual en Tortosa, cerca de la desembocadura, es de $431 \mathrm{~m}^{3} \mathrm{~s}^{-1}$, que corresponde a una aportación hídrica anual de $13.812 \mathrm{hm}^{3}\left(\sigma=5474 \mathrm{hm}^{3} \mathrm{a}^{-1}\right.$, donde $\sigma$ es la desviación estándar de la serie 1912-2018). El caudal medio mínimo anual se ha reducido desde algo más de $600 \mathrm{~m}^{3} \mathrm{~s}^{-1}$, en el período 1912-1930, hasta algo menos de $300 \mathrm{~m}^{3} \mathrm{~s}^{-1}$ durante las dos primeras décadas del siglo XXI. La aportación hídrica varía sustancialmente, desde una aportación máxima anual de $30.821 \mathrm{hm}^{3}$ (1914-1915) hasta un mínimo de $4284 \mathrm{hm}^{3}$ (1989-1990). El caudal máximo instantáneo se estimó en ca. $12.000 \mathrm{~m}^{3} \mathrm{~s}^{-1}$ en Tortosa durante la riada de 1907 (Novoa, 1984). Las crecidas de mayor magnitud se han registrado en 1787, 1907 y 1866 con caudales de 12.900, 12.000 y $7750 \mathrm{~m}^{3} \mathrm{~s}^{-1}$, respectivamente (Balasch et al., 2019). Hoy en día, 133 embalses regulan alrededor de $2 / 3$ de la aportación media anual de la cuenca (i.e. $8000 \mathrm{hm}^{3}$ ). Los 25 embalses más grandes de la cuenca $\left(>50 \mathrm{hm}^{3}\right)$ representan el $90 \%$ de la capacidad total de almacenamiento de la cuenca. El complejo de presas más grande de la cuenca se encuentra en el tramo bajo del río Ebro, y está constituido por las presas de Mequinenza (construida en 1966, $1534 \mathrm{hm}^{3}$ ), Ribarroja $\left(1969,207 \mathrm{hm}^{3}\right)$ y Flix $\left(1948,11 \mathrm{hm}^{3}\right)$, cerrando en este punto el $97 \%$ del área de la cuenca (Fig. 1B).

Este estudio se centra en el embalse de Ribarroja. El Ebro aguas arriba del embalse de Mequinenza drena una cuenca de aproximadamente $51.210 \mathrm{~km}^{2}$, siendo el del Ebro el mayor embalse $\left(500 \mathrm{hm}^{3}\right)$ aguas arriba de este punto (Índice de Regulación IR =0,77; ver Batalla et al., 2004). La aportación media anual al embalse de Mequinenza es de $7174 \mathrm{hm}^{3}$ (periodo 1973-2016). Los principales afluentes al embalse de Ribarroja por el margen izquierdo (Pirineos) son el Segre y el Cinca, mientras que los del margen derecho (Sistema Ibérico) son el Matarraña y el Algars.

Los ríos Segre y Cinca son los afluentes más importantes del Ebro, drenando cuencas de $12.751 \mathrm{~km}^{2}$ y $9608 \mathrm{~km}^{2}$, respectivamente. La aportación hídrica de estos dos ríos al Ebro es la más importante de la cuenca (5122 $\mathrm{hm}^{3}$ año de promedio, series 1949-2016), y dado su régimen nivo-pluvial (alta acumulación de nieve en el Pirineo central durante los meses invernales) presenta un máximo en primavera y caudales relativamente constantes en verano. Ambas cuencas están fuertemente reguladas por grandes presas, con una capacidad total de agua embalsada de $2120 \mathrm{hm}^{3}$ en el caso del Segre y $993 \mathrm{hm}^{3}$ en el caso del Cinca ( $\mathrm{IR}=1,49$ y 0,59 respectivamente). Los afluentes mediterráneos, Matarraña y Algars, se caracterizan por un régimen hidrológico fuertemente influenciado por la precipitación, registrando máximos en primavera y otoño, y el mínimo en verano. El área de la cuenca del Matarraña es de $1717 \mathrm{~km}^{2}$, de los cuales $405 \mathrm{~km}^{2}$ corresponden a su principal afluente, el Algars. Dentro de la cuenca del Matarraña, únicamente el río Pena, un pequeño afluente en la cabecera, está regulado por la presa del mismo nombre con una capacidad de $17,8 \mathrm{hm}^{3}(\mathrm{IR}=1,13)$. La aportación media anual de la cuenca del Matarraña es de $35 \mathrm{hm}^{3}$ (periodo 1974-2016). La cuenca del río Algars no está regulada por embalses, por lo tanto, el IR es 0 . Su aportación media anual es de $21.2 \mathrm{hm}^{3}$ (periodo 1974-2016). 


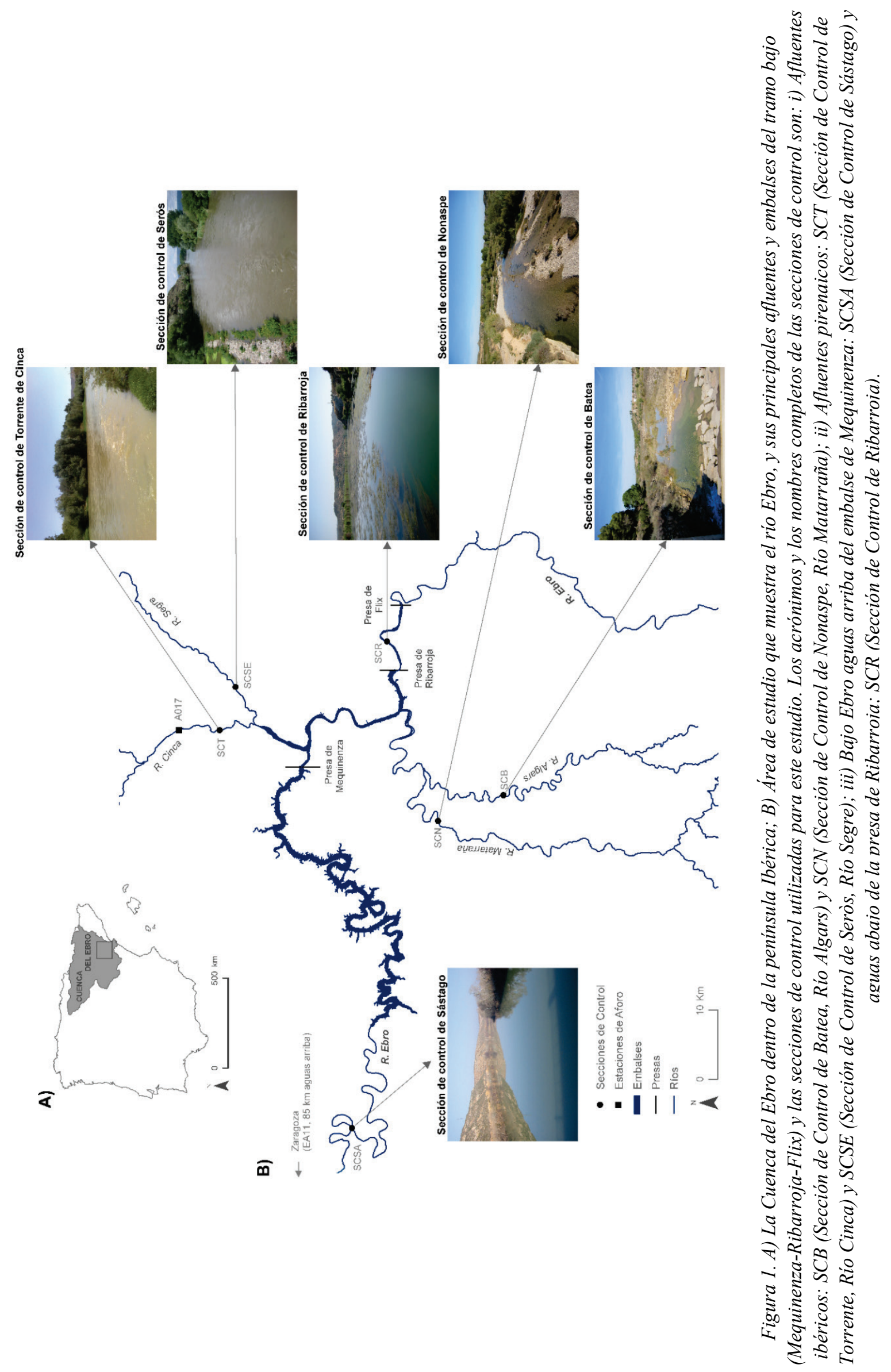




\section{Materiales y métodos}

Los datos utilizados en este estudio consisten en series continuas de caudal y turbidez recogidas entre 2008 y 2011 en diferentes secciones de control, a la entrada y salida del embalse de Ribarroja y en los principales afluentes que desembocan en este embalse. Para el particular caso de la Sección de Control de Sástago (SCSA, entrada al embalse de Mequinenza), se ha utilizado una relación estadística entre caudal y sedimento en suspensión obtenido a partir de muestras discretas durante el periodo 20032004. El análisis de toda la información de manera conjunta permite construir un balance de sedimentos del embalse de Ribarroja (para la localización de todas las secciones de control ver Fig. 1B). A continuación, se presentan los detalles metodológicos específicos.

\subsection{Caudal}

Las series de caudal en continuo (en adelante $Q$ ) se han obtenido de tres maneras diferentes: i) a partir de estaciones de aforo propiedad de Confederación Hidrográfica del Ebro (CHE); ii) a partir de secciones de control sedimentométrico propias (Grupo RIUS-UdL) por medio de sensores de nivel de agua; y iii) a partir del tránsito del caudal desde estaciones de aforo cercanas. Los detalles de las tres fuentes de datos se dan a continuación:

i) Las estaciones de medición de CHE se etiquetan como sección de control de Batea (SCB), sección de control de Nonaspe (SCN) y sección de control de Serós (SCSE) (ver la Figura 1B para localización de las secciones de control). En estas estaciones, se mide el nivel del agua (h) con una frecuencia quinceminutal por medio de un limnígrafo OTT®, y posteriormente se transforma en $Q$ mediante una curva de gasto $h / Q$ específica.

ii) La sección de control propia donde se registra el nivel de agua en continuo es la sección de control de Torrente de Cinca (SCT, ver Fig. 1B). En esta sección, el nivel de agua se midió mediante un TruTrack ${ }^{\circledR}$ WT-HR (sensor capacitivo de nivel de agua con registrador de datos integrado). El error promedio del sensor, estimado a partir de la comparación de mediciones directas en una escala y las lecturas del sensor durante las visitas de campo semanales era de un 2\% (Tena y Batalla, 2013). El nivel de agua se registró a intervalos de 15 minutos. En esta sección, $Q$ se calculó utilizando el método de nivel / área mediante el modelo 1D WinXSPRO ${ }^{\circledR}$. Este software calcula el caudal mediante la ecuación de Manning, a partir de una sección topográfica y la distribución granulométrica representativa del tramo a estudiar, necesaria para estimar el coeficiente de rugosidad $(n)$. Además, para corroborar los valores obtenidos mediante este método, se estableció una relación entre el nivel de agua en SCT y el caudal, obtenida en la estación de aforo más cercana (Fraga EA017; ver Fig. 1B). Una vez conocidos los parámetros hidráulicos (por ejemplo, tiempo de tránsito, atenuación/laminación de caudal), los hidrogramas de las estaciones oficiales se transitaron a SCT, siguiendo el método de Muskingum para toda la serie de datos (Shaw, 1983). La desviación entre los caudales transitados y los caudales modelados fuer inferior al 5\%.

iii) En el caso de la sección de control de Sástago (SCSA) y la sección de control de Ribarroja (SCR, ver Fig. 1B), donde no había sensores de nivel, se ha seguido un procedimiento similar al descrito en ii). En ambos casos, los caudales se han transitado directamente desde las estaciones de medición más cercanas, también mediante el método de Muskingum. En SCR, los caudales se transitaron desde la presa de Ribarroja (datos facilitados por la CHE), ubicada $3 \mathrm{~km}$ aguas arriba. En el caso de SCSA, el caudal se transitó desde la estación de aforo oficial EA11 de Zaragoza (ubicada a $85 \mathrm{~km}$ aguas arriba), teniendo en cuenta que las aportaciones entre estas dos secciones no son significativas (Vericat y Batalla, 2006). 


\subsection{Sedimento en suspensión}

La concentración de sedimentos en suspensión (CSS) se obtuvo a partir de los registros continuos de turbidez. Los datos de turbidez se obtuvieron de tres fuentes diferentes: i) de las estaciones oficiales de calidad del agua de la Agencia Catalana del Agua (ACA), ii) de las secciones de control equipadas con sondas de turbidez propias (Grupo RIUS-UdL; ver la Fig. 1B para más detalles), y iii) a partir de relaciones estadísticas entre el caudal $(Q)$ y el transporte de sedimentos en suspensión (CSS) ( $\tan$ solo en SCSA). Específicamente:

i) Las estaciones de calidad del agua operadas por el ACA. Estos datos (en SCSE) se registran cada 15 minutos por medio de una sonda de turbidez Campbell® OBS-3 + (0-4000 NTU).

ii) En las secciones de control propias ( $\mathrm{SCR}, \mathrm{SCB}, \mathrm{SCN}$ y $\mathrm{SCT}$ ), se registra la turbidez en continuo. En estas secciones, la turbidez se midió mediante turbidímetros ópticos McVann ${ }^{\circledR}$ Analite. Sin embargo, dependiendo de las concentraciones de sedimentos en suspensión estimadas para cada río, se utilizaron modelos diferentes. Por ejemplo, en aquellas secciones aguas abajo de las presas (SCR), donde se esperaba que las concentraciones fueran bajas, se utilizó el NEP9510, una sonda de turbidez de bajo rango (0-1000 NTU). Por otro lado, en los afluentes (SCB, SCN y SCT), donde se esperaba una mayor concentración de sedimentos, se instalaron sondas de turbidez de mayor rango, NEP9530 (0-3000 NTU). El almacenamiento de los datos de turbidez se realizaba en data-loggers Campbell® (CR-510, CR-800 y CR1000) con una frecuencia quinceminutal, obtenidos a partir de los promedios de las lecturas que el instrumento realizaba cada 5 segundos. Con el propósito de evitar problemas de variabilidad y agilizar los cálculos, se utilizaron los promedios horarios.

iii) Aguas arriba de la presa de Mequinenza no se disponía de un turbidímetro. Así, para estimar la carga anual de sedimentos en suspensión en SCSA y poder cerrar el balance sedimentario en Ribarroja se aplicaron las relaciones entre $Q$ y $C S S$ obtenidas previamente por Vericat y Batalla (2006), utilizando el hidrograma transitado previamente. Se utilizó una relación estadísticamente significativa $Q$ - CSS obtenida por Vericat y Batalla (2006) para el año hidrológico 2003-04 para estimar las concentraciones de sedimentos suspensión, y posteriormente las cargas sólidas totales. El año 2003-04 fue similar desde el punto de vista hidrológico al período de estudio 2008-2011, por lo que se considera un modelo representativo del período de estudio. En este caso se trataba de tener un valor de referencia, puesto que como se ha visto en trabajos realizados previamente en este tramo (Roura, 2004; Vericat y Batalla, 2006), el embalse de Mequinenza puede retener hasta el $95 \%$ de los sedimentos en suspensión transportados anualmente desde aguas arriba.

Como parte del programa de muestreo de sedimentos en suspensión implementado desde 2002 en el tramo bajo del río Ebro y sus principales afluentes, se recogieron muestras manuales de 1 litro de manera intensiva durante eventos de crecida y de forma rutinaria (semanal o quincenal) durante períodos de caudales bajos (para más detalles, consultar Vericat y Batalla, 2005, 2006; Tena et al. 2011, 2012; Tena y Batalla, 2013). En total, se utilizaron 737 muestras de sedimentos en suspensión para calibrar los datos de turbidez y complementar los cálculos de cargas sedimentarias. Las muestras se filtraron al vacío por medio de filtros de microfibra de vidrio (Filter-Lab ${ }^{\circledR}$, tamaño de poro de $1,2 \mu \mathrm{m}$ ), se secaron y se pesaron en el laboratorio para determinar la concentración de sedimento en suspensión (CSS en $\mathrm{mg}^{-1}$ ). El contenido de materia orgánica se determinó siguiendo los métodos resumidos por Tena et al. (2011). Una vez que se determinó el contenido en materia orgánica, se restó del peso del filtro. Posteriormente, se estableció una correlación entre NTU y CSS para cada sonda (sección de control). En la mayoría de los casos, la relación siguió una regresión lineal (CSS $=a \times N T U+b$, donde $a$ varía de 0,6 a 0,9 y $b$ de -6 a 3,6, con un coeficiente de determinación $\left(\mathrm{r}^{2}\right)$ entre 0,89 y 0,98$)$. Solo en SCSE la relación entre NTU y CSS siguió una relación del tipo CSS $=0,0093 \times N T U^{2}+1,1303 \times N T U+3,8$ 
$\left(\mathrm{r}^{2}=0,96\right)$. Finalmente, la carga de sedimento en suspensión se calculó multiplicando la CSS (en mg $\mathrm{l}^{-}$ ${ }^{1}$, determinada a partir de la turbidez) y el $Q$ continuo $\left(\mathrm{en}^{3} \mathrm{~s}^{-1}\right)$.

\section{Resultados}

\subsection{Hidrología}

Antes de entrar en el embalse de Mequinenza en la sección de control de Sástago (SCSA), el río Ebro ha recibido ya el aporte de muchos de sus afluentes, aunque todavía no el de los más importantes desde el punto de vista hidro-sedimentario. En SCSA, el aporte medio anual durante los 3 años de estudio fue de $5927 \mathrm{hm}^{3}\left(\sigma=1660 \mathrm{hm}^{3}\right)$ un valor inferior a $\operatorname{los} 7310 \mathrm{hm}^{3}\left(\sigma=2690 \mathrm{hm}^{3}\right)$ registrados históricamente (1912-2016) y, por lo tanto, el período de estudio puede considerarse como seco. El caudal medio $\left(Q_{\mathrm{med}}\right)$ en SCSA para el período de estudio fue de $188 \mathrm{~m}^{3} \mathrm{~s}^{-1}\left(\sigma=201 \mathrm{~m}^{3} \mathrm{~s}^{-1}\right)$, oscilando entre $19 \mathrm{~m}^{3} \mathrm{~s}^{-1}$ y $1545 \mathrm{~m}^{3} \mathrm{~s}^{-1}$, éste último alcanzado durante una crecida en febrero de 2009 (equivalente a un período de retorno de 1,2 años, Tabla 1). El coeficiente de variación interanual (es decir, $\mathrm{CV}=Q_{\sigma} /$ $Q_{\mathrm{med}}$ ) fue del $107 \%$, un valor que confirma la alta variabilidad temporal.

El río Segre, tras recoger las aguas de los grandes afluentes del Pirineo, se convierte en el afluente más importante del Ebro. Pocos kilómetros antes de su entrada en el embalse de Ribarroja, confluye con el río Cinca, su principal afluente. La aportación media anual en la sección de control de Seròs (SCSE, río Segre) durante los 3 años del período de estudio fue de $1427 \mathrm{hm}^{3}\left(\sigma=430 \mathrm{hm}^{3}\right)$, un valor ligeramente inferior a los $1676 \mathrm{hm}^{3}\left(\sigma=189 \mathrm{hm}^{3}\right)$ registrados en la sección de control de Torrente (SCT) en el río Cinca. En ambos casos, la escorrentía está muy por debajo de los $5122 \mathrm{hm}^{3} \mathrm{a}^{-1}(\sigma=1985$ $\mathrm{hm}^{3}$ ) registrados para el sistema Segre-Cinca desde 1950 hasta la actualidad, confirmando que se trató de un periodo seco. Además, la proporción de agua producida en las dos cuencas (45 y 55\% en SCSE y $\mathrm{SCT}$, respectivamente) difiere de la relación histórica, que generalmente es inversa, 55\% en SCSE y $45 \%$ en SCT. El cambio en la proporción de escorrentía relativa entre los dos ríos puede estar relacionado con el incremento reciente de la regulación del caudal en el Segre (Batalla et al., 2004). El $Q_{\text {med }}$ en SCSE fue de $45 \mathrm{~m}^{3} \mathrm{~s}^{-1}\left(\sigma=36 \mathrm{~m}^{3} \mathrm{~s}^{-1}\right)$, con un caudal mínimo de $13 \mathrm{~m}^{3} \mathrm{~s}^{-1} \mathrm{y}$ un caudal máximo de $498 \mathrm{~m}^{3} \mathrm{~s}^{-1}$ (valor que representa un período de retorno de 2 años) alcanzado durante una crecida en la primavera de 2010. El $Q_{\text {med }}$ en SCT fue de $53 \mathrm{~m}^{3} \mathrm{~s}^{-1}\left(\sigma=36 \mathrm{~m}^{3} \mathrm{~s}^{-1}\right)$, y osciló entre $15 \mathrm{~m}^{3} \mathrm{~s}^{-1}$ y 593 $\mathrm{m}^{3} \mathrm{~s}^{-1}$, crecida con un período de retorno de 1,2 años (ver Tabla 1 para obtener información hidrológica completa). El coeficiente de variación interanual fue del 79\% en SCSE y del 68\% en SCT.

Los afluentes que fluyen al embalse de Ribarroja desde el Sistema Ibérico muestran unas características más secas que los pirenaicos. El río Algars se une al Matarraña $22 \mathrm{~km}$ antes de su desembocadura en el embalse de Ribarroja. Las cabeceras de estos dos ríos se encuentran en el mismo macizo montañoso, por lo que sus características hidroclimáticas son muy similares. Al igual que en el caso de los afluentes pirenaicos, los datos reflejan el carácter seco del período de estudio (2008-2011). La aportación hídrica durante el período de estudio fue de $16 \mathrm{hm}^{3} \mathrm{a}^{-1}\left(\sigma=9,6 \mathrm{hm}^{3}\right)$ en la sección de control de Nonaspe (SCN, Matarraña) y $12 \mathrm{hm}^{3} \mathrm{a}^{-1}\left(\sigma=4,6 \mathrm{hm}^{3}\right)$ en la sección de control de Batea (SCB, Algars), estando ambos valores muy por debajo de los $38 \mathrm{hm}^{3} \mathrm{a}^{-1}\left(\sigma=39 \mathrm{hm}^{3}\right)$ y $21 \mathrm{hm}^{3} \mathrm{a}^{-1}\left(\sigma=17 \mathrm{hm}^{3}\right)$ registrados históricamente (desde 1974 hasta la actualidad) (ver Tabla 1). El $Q_{\text {med }}$ en SCN fue de $0,5 \mathrm{~m}^{3} \mathrm{~s}^{-1}\left(\sigma=1,9 \mathrm{~m}^{3} \mathrm{~s}^{-1}\right)$, mientras que en SCB fue $\sigma 0,4 \mathrm{~m}^{3} \mathrm{~s}^{-1}\left(\sigma=1,3 \mathrm{~m}^{3} \mathrm{~s}^{-1}\right)$. El caudal fue especialmente bajo durante los meses de verano, pudiendo encontrarse el cauce seco en ambas secciones durante periodos más o menos largos (7 y 23\% del tiempo en SCN y SCB, respectivamente). El caudal máximo en SCN fue de $50 \mathrm{~m}^{3} \mathrm{~s}^{-1}$ y se alcanzó en octubre de 2011 (con un período de retorno de 1,6 años), mientras que en SCB fue de $39,5 \mathrm{~m}^{3} \mathrm{~s}^{-1} \mathrm{y}$ ocurrió en marzo de 2011 (período de retorno de 1,7 años). La variabilidad interanual fue notable en comparación con los afluentes pirenaicos, alcanzando un $\mathrm{CV}$ de $383 \%$ en $\mathrm{SCN}$ y $357 \%$ en SCB. En estos ríos mediterráneos, las crecidas son las principales responsables de la aportación hídrica de la cuenca. 
Tabla 1 Caudales para el periodo de estudio (2008-2011) en las diferentes secciones de estudio del Ebro y sus principales afluentes (ver Fig. IB para la ubicación y abreviación de las secciones)

\begin{tabular}{|c|c|c|c|c|c|c|c|}
\hline Área & Sección & Año & $\begin{array}{c}Q_{\text {med }} \\
\left(\mathbf{m}^{3} \mathbf{s}^{-1}\right)\end{array}$ & $\underset{\left(\mathbf{m}^{3} \mathbf{s}^{-1}\right)}{Q_{\max }}$ & $\begin{array}{c}Q_{\min } \\
\left(\mathbf{m}^{3} \mathbf{s}^{-1}\right)\end{array}$ & $\begin{array}{c}\sigma^{1} \\
\left(m^{3} s^{-1}\right)\end{array}$ & $\begin{array}{c}\text { Aportación } \\
\left(\mathrm{hm}^{3}\right)\end{array}$ \\
\hline \multirow{8}{*}{ 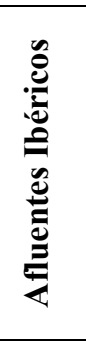 } & \multirow{4}{*}{ 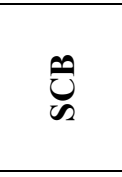 } & 2008-09 & 0,5 & 23,1 & 0,0 & 1,0 & 15,4 \\
\hline & & $2009-10$ & 0,2 & 5,0 & 0,0 & 0,4 & 6,5 \\
\hline & & $2010-11$ & 0,4 & 39,5 & 0,0 & 2,0 & 13,1 \\
\hline & & $2008-11$ & 0,4 & 39,5 & 0,0 & 1,3 & $11,7^{*}$ \\
\hline & \multirow{4}{*}{$Z_{\infty}^{Z}$} & 2008-09 & 0,8 & 32,8 & 0,2 & 1,9 & 26,0 \\
\hline & & $2009-10$ & 0,2 & 3,9 & 0,0 & 0,4 & 7,0 \\
\hline & & $2010-11$ & 0,5 & 49,9 & 0,0 & 2,7 & 14,7 \\
\hline & & 2008-11 & 0,5 & 49,9 & 0,0 & 1,9 & $15,9^{*}$ \\
\hline \multirow{8}{*}{ 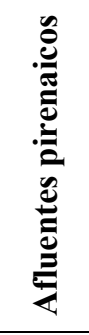 } & \multirow{4}{*}{ t) } & 2008-09 & 54,0 & 230,6 & 18,3 & 35,2 & 1702,0 \\
\hline & & $2009-10$ & 58,7 & 593,2 & 15,0 & 48,2 & 1851,3 \\
\hline & & 2010-11 & 46,8 & 127,4 & 17,6 & 17,3 & 1475,9 \\
\hline & & $2008-11$ & 53,2 & 593,2 & 15,0 & 36,2 & $1676,4^{*}$ \\
\hline & \multirow{4}{*}{ 点 } & 2008-09 & 58,0 & 245,3 & 19,0 & 30,5 & 1829,3 \\
\hline & & $2009-10$ & 46,8 & 498,2 & 12,8 & 48,5 & 1477,1 \\
\hline & & $2010-11$ & 30,9 & 114,0 & 13,8 & 13,7 & 973,4 \\
\hline & & $2008-11$ & 45,2 & 498,2 & 12,8 & 35,8 & $1426,6^{*}$ \\
\hline \multirow{8}{*}{ 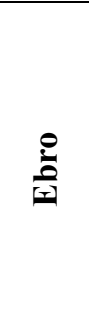 } & \multirow{4}{*}{ 依 } & 2008-09 & 234,2 & 1544,7 & 36,9 & 252,1 & 7384,8 \\
\hline & & $2009-10$ & 199,0 & 1476,4 & 36,5 & 190,8 & 6275,1 \\
\hline & & 2010-11 & 130,7 & 932,4 & 19,1 & 126,6 & 4121,1 \\
\hline & & $2008-11$ & 187,9 & 1544,7 & 19,1 & 201,3 & $5927,0^{*}$ \\
\hline & \multirow{4}{*}{ 萬 } & $2008-09$ & 344,3 & 1259,5 & $1,8^{* *}$ & 251,3 & 10859,4 \\
\hline & & 2009-10 & 314,1 & 2028,3 & $0,8^{* *}$ & 198,7 & 9904,9 \\
\hline & & $2010-11$ & 214,9 & 1123,1 & $1,5^{* *}$ & 119,5 & 6775,7 \\
\hline & & $2008-11$ & 291,1 & 2028,3 & $0,8^{* *}$ & 205,0 & $9180,0^{*}$ \\
\hline
\end{tabular}

\footnotetext{
${ }^{1}$ Desviación estándar.

${ }^{*}$ Valor medio.

** Valor mínimo cuando la presa de Ribarroja estaba cerrada.
}

En la sección de control de Ribarroja (SCR; ver la Fig. 1B para la ubicación exacta), el impacto que ejerce el complejo de presas de Mequinenza-Ribarroja (CPMR en adelante) en la hidrología es notable. La aportación media anual en este punto durante el período de estudio fue de $9180 \mathrm{hm}^{3}$. El caudal medio en esta sección fue de $291 \mathrm{~m}^{3} \mathrm{~s}^{-1}\left(\sigma=205 \mathrm{~m}^{3} \mathrm{~s}^{-1}\right)$, mostrando una variabilidad interanual más baja $(73 \%)$ que las mencionadas estaciones aguas arriba. El caudal máximo en este punto se registró en enero de 2010 durante una crecida natural en la que el caudal máximo liberado por la presa de Ribarroja alcanzó $2028 \mathrm{~m}^{3} \mathrm{~s}^{-1}$, un caudal equivalente a un periodo de retorno de 4 años. Se entiende por crecida natural la que se genera en la cuenca como consecuencia de un episodio lluvioso y que los embalses transitan, en contraposición a las crecidas artificiales que con fines ambientales se generan desde las presas (Batalla y Vericat, 2009).

La dinámica temporal de las aportaciones hídricas en las diferentes secciones de control se ha estudiado mediante un análisis de frecuencias. Los resultados se presentan en diagramas de cajas y bigotes o bloxplots (Fig. 2A). Este método permite conocer el porcentaje de tiempo necesario para transportar distintas fracciones de la aportación anual. Se han elegido un total de 7 percentiles característicos de la aportación anual: 5, 16, 25, 50, 75, 84 y 95\%.

La Figura 2A muestra el tiempo necesario para alcanzar diferentes percentiles característicos de la aportación hídrica media anual. El 50\% de la aportación hídrica se efectúa con un porcentaje de tiempo distinto en los afluentes ibéricos respecto a los pirenaicos y el propio Ebro. En SCB y SCN se requiere del 4 y del 3\% del tiempo para transportar el 50\% de la aportación, mientras que en SCT y SCSE se requiere alrededor del $25 \%$ del tiempo, valores superiores a los requeridos por SCSA y SCR (aproximadamente el 15 y $20 \%$ del tiempo, respectivamente). Estas diferencias muestran el marcado 
carácter mediterráneo de los afluentes ibéricos, en los que la aportación estaría controlada principalmente por las crecidas, frente a los caudales más regulares de los afluentes pirenaicos y del propio Ebro.

Si tomamos como referencia los percentiles más elevados, se puede observar cómo hay parte del tiempo en el que la aportación de los afluentes ibéricos es muy baja o incluso nula. Estos ríos tan solo requieren del $40 \%$ del tiempo o menos para transportar el $95 \%$ de la aportación. En el caso de los afluentes pirenaicos y del propio Ebro, en el que los caudales son más constantes, el tiempo necesario para transportar el $95 \%$ de la aportación en todos los casos es superior al $85 \%$ del tiempo.

\subsection{Transporte de sedimentos}

\subsubsection{Transporte de sedimentos en suspensión}

Las concentraciones de sedimentos en suspensión en la sección de control de SCSA, aguas arriba del embalse Mequinenza, son elevadas comparadas con la estación aguas abajo de la presa de Ribarroja (SCR). Los valores registrados en Sástago son la consecuencia de una menor regulación de este tramo del río y, sobre todo, de la aportación de destacados afluentes como el Gállego y el Jalón. La concentración media de sedimentos en suspensión $\left(C S S_{\mathrm{med}}\right)$ para el período de estudio fue de $56 \mathrm{mg} \mathrm{l}^{-1}$ $\left(\sigma=55,4 \mathrm{mg} \mathrm{l}^{-1}\right)$, mientras que la máxima $\left(C S S_{\max }\right)$ fue alrededor de $510 \mathrm{mg} \mathrm{l}^{-1}$ (ver Tabla 2 para el resumen completo de las CSS registradas)

Tabla 2 Datos de sedimento en suspensión para el periodo de estudio (2008-2011) en las diferentes secciones de estudio del Ebro y sus principales afluentes (ver Fig. 1B para la ubicación y abreviación de las secciones).

\begin{tabular}{|c|c|c|c|c|c|c|c|c|}
\hline Área & Sección & Año & $\begin{array}{l}\mathrm{CSS}_{\text {med }} \\
\left(\mathrm{mg} \mathrm{l}^{-1}\right)\end{array}$ & $\begin{array}{l}\mathrm{CSS}_{\max } \\
\left(\mathrm{mg} \mathrm{l}^{-1}\right)\end{array}$ & $\begin{array}{l}\mathrm{CSS}_{\min } \\
\left(\mathrm{mg} \mathrm{l}^{-1}\right)\end{array}$ & $\begin{array}{c}\sigma^{1} \\
\left(\mathrm{mg} \mathrm{l}^{-1}\right)\end{array}$ & $\begin{array}{c}\text { Carga Total } \\
\left(10^{6} t\right)\end{array}$ & $\begin{array}{l}\text { Producción } \\
\left(\mathrm{t} \mathrm{km}^{2} \mathrm{yr}^{-1}\right)\end{array}$ \\
\hline \multirow{8}{*}{ 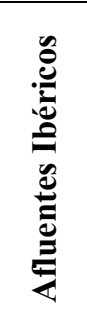 } & \multirow{4}{*}{ 己ै } & 2008-09 & 9,7 & 632,9 & 0 & 14,4 & 0,00024 & 0,7 \\
\hline & & $2009-10$ & 6,9 & 261,7 & 0,1 & 7,4 & 0,00007 & 0,2 \\
\hline & & $2010-11$ & 10 & 510 & 0,4 & 13,1 & 0,00053 & 1,6 \\
\hline & & $2008-11$ & 8,9 & 632,9 & 0 & 12,1 & $0,00083\left(0,00028^{*}\right)$ & 0,9 \\
\hline & \multirow{4}{*}{ Z } & 2008-09 & 4,5 & 309,3 & 0,4 & 13 & 0,00064 & 0,6 \\
\hline & & $2009-10$ & 3,2 & 46 & 0,4 & 2,5 & 0,00003 & 0 \\
\hline & & $2010-11$ & 3,9 & 280,7 & 0,7 & 10,1 & 0,00075 & 0,7 \\
\hline & & $2008-11$ & 3,8 & 309,3 & 0,4 & 9,6 & $0,00143\left(0,00048^{*}\right)$ & 0,5 \\
\hline \multirow{8}{*}{ 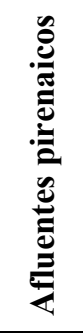 } & \multirow{4}{*}{ 记 } & 2008-09 & 80,4 & 2033 & 7,5 & 167,1 & 0,15 & 16 \\
\hline & & $2009-10$ & 50,7 & 1701,5 & 8,8 & 81,1 & 0,14 & 15 \\
\hline & & $2010-11$ & 35,4 & 1434,5 & 6,7 & 71 & 0,07 & 6,9 \\
\hline & & $2008-11$ & 55,5 & 2033 & 6,7 & 116,3 & $0,365\left(0,12^{*}\right)$ & 12,7 \\
\hline & \multirow{4}{*}{ 己 } & $2008-09$ & 26,6 & 486,5 & 6,1 & 27,9 & 0,06 & 4,5 \\
\hline & & $2009-10$ & 25 & 1551,7 & 7,2 & 38 & 0,05 & 3,7 \\
\hline & & $2010-11$ & 16,9 & 222 & 6,1 & 9,7 & 0,02 & 1,4 \\
\hline & & $2008-11$ & 22,8 & 1551,7 & 6,1 & 28,1 & $0,121\left(0,040^{*}\right)$ & 3,2 \\
\hline \multirow{8}{*}{$\stackrel{\stackrel{0}{0}}{\text { 国 }}$} & \multirow{4}{*}{$\underset{\mathscr{\infty}}{\mathbb{\infty}}$} & 2008-09 & 69,5 & 510 & 16,5 & 73,3 & 1,07 & 20,9 \\
\hline & & 2009-10 & 55,8 & 484,8 & 16,3 & 48,8 & 0,62 & 12,1 \\
\hline & & $2010-11$ & 42,5 & 283,5 & 9 & 33 & 0,29 & 5,6 \\
\hline & & $2008-11$ & 55,9 & 510 & 9 & 55,4 & $1,978\left(0,66^{*}\right)$ & 12,9 \\
\hline & \multirow{4}{*}{$\underset{\mathscr{U}}{\tilde{U}}$} & 2008-09 & 5,5 & 72,4 & 0 & 4,2 & 0,06 & 0,7 \\
\hline & & $2009-10$ & 5,4 & 214,2 & 1,4 & 5,7 & 0,05 & 0,6 \\
\hline & & $2010-11$ & 3,6 & 206,2 & 0,2 & 4,6 & 0,02 & 0,3 \\
\hline & & $2008-11$ & 4,9 & 214,2 & 0 & 4,9 & $0,131\left(0,043^{*}\right)$ & 0,5 \\
\hline
\end{tabular}

${ }^{1}$ Desviación estándar.

*Valor medio. 
En el caso de los afluentes pirenaicos, la $C S S_{\text {med }}$ observada fue generalmente mayor que la de los afluentes ibéricos. La CSS med para el período de estudio en el río Segre (SCSE) fue de $23 \mathrm{mg}^{-1}$ ( $\sigma=$ $\left.28 \mathrm{mg} \mathrm{l}^{-1}\right)$, un valor inferior a los $56 \mathrm{mg} \mathrm{l}^{-1}\left(\sigma=116 \mathrm{mg} \mathrm{l}^{-1}\right)$ registrados en el Cinca (SCT). La CSS $S_{\max }$ también fue mayor en SCT que en SCSE (2033 mg $\mathrm{l}^{-1}$ y $1552 \mathrm{mg} \mathrm{l}^{-1}$, respectivamente). Por su parte, los ríos del Sistema Ibérico mostraron una $C_{S S} S_{\mathrm{med}}$ de $9 \mathrm{mg} \mathrm{l}^{-1}$ en el Algars (SCB, $\sigma=12 \mathrm{mg}^{1-1}$ ), y $4 \mathrm{mg} \mathrm{l}^{-1}$ en el Matarraña ( $\mathrm{SCN}, \sigma=10 \mathrm{mg} \mathrm{l}^{-1}$ ). La CSS $S_{\max }$ también fue mayor en SCB, con un pico de sedimento de $633 \mathrm{mg} \mathrm{l}^{-1}$, dos veces superior al pico registrado en SCN (Tabla 2). La CSS $S_{\max }$ se alcanzó en ambos ríos durante el año 2008-09, pero bajo diferentes condiciones de caudal. Mientras que en el Matarraña coincidió con el caudal máximo $\left(32 \mathrm{~m}^{3} \mathrm{~s}^{-1}\right)$, en el caso del Algars ocurrió durante una crecida de baja magnitud $\left(0,75 \mathrm{~m}^{3} \mathrm{~s}^{-1}\right)$, lo que muestra un claro ejemplo de no linealidad en las relaciones Q-CSS en cuencas donde el suministro y la disponibilidad de sedimentos es variable.

El efecto del complejo de embalses de Mequinenza y Ribarroja se hace patente en el transporte de sedimento en suspensión. La sección de control de Ribarroja, localizada aguas abajo del embalse de Ribarroja, registró CSS de un orden de magnitud inferior a los valores estimados aguas arriba de Mequinenza. Como se indica en la Tabla 2, la $C_{S S}$ med en SCR no superó los $5 \mathrm{mg} \mathrm{l}^{-1}$, mostrando una variabilidad muy baja entre los tres años de estudio $\left(\sigma=2 \mathrm{mg} \mathrm{l}^{-1}\right)$. La CSS $\max$ tuvo lugar durante una crecida de mantenimiento realizada en mayo de 2010, llegando a alcanzar los $214 \mathrm{mg} \mathrm{l}^{-1}$ (Batalla y Vericat, 2009).

\subsubsection{Análisis de frecuencias}

Al igual que en el caso de la aportación hídrica, las cargas sedimentarias y su dinámica temporal se han estudiado en las diferentes secciones de control mediante un análisis de frecuencias (Fig. 2B).

Se distinguen diferentes patrones según el régimen hidrológico del río y el grado de regulación de cada sección. La carga de sedimentos en los afluentes pirenaicos se muestra relativamente constante en el tiempo. La Figura 2B indica que el $84 \%$ del sedimento fue transportado en aproximadamente el $65 \%$ del tiempo en el río Cinca (correspondiente a un $Q>35 \mathrm{~m}^{3} \mathrm{~s}^{-1}$ ); mientras que en el Segre se transportó la misma proporción en aproximadamente $2 / 3$ del tiempo (para $Q>29 \mathrm{~m}^{3} \mathrm{~s}^{-1}$ ). Las crecidas y los caudales altos (tomados como el doble del caudal medio) fueron responsables del 43 y el $52 \%$ de la carga de sedimentos transportada durante el período de estudio en el Segre y el Cinca, respectivamente.

En el caso de los afluentes del Sistema Ibérico (Fig. 2B), el 84\% del sedimento se transportó en el $9 \%$ del tiempo $\left(Q>0,6 \mathrm{~m}^{3} \mathrm{~s}^{-1}\right)$ en el río Algars, y en menos del $2 \%$ en el caso del Matarraña $(Q>$ $\left.3,8 \mathrm{~m}^{3} \mathrm{~s}^{-1}\right)$. En estos dos ríos, la carga está más dominada por las crecidas, que pueden transportar hasta el $95 \%$ de la carga anual. Por ejemplo, si se analizan las frecuencias para cada año de manera independiente, se observa que en 2009 y 2011 las crecidas transportaron el 95\% de la carga en los ríos del Sistema Ibérico. En general, los diagramas están comprimidos en rangos inferiores cuanto más secos son los ambientes (como es el caso de las cuencas mediterráneas del Sistema Ibérico); como se ha indicado, en estas cuencas, las crecidas son responsables de transportar una porción más grande de sedimentos que en otras cuencas con regímenes hidroclimáticos más húmedos.

De las dos secciones localizadas en el curso principal del Ebro, la de SCSA es la que presenta una mayor variabilidad temporal en la carga. Los diagramas de cajas (Fig. 2B) muestran que el 84\% del sedimento en suspensión fue transportado en el $25 \%$ del tiempo $\left(Q>174 \mathrm{~m}^{3} \mathrm{~s}^{-1}\right)$, mostrando una variabilidad intermedia entre las cuencas ibéricas y pirenaicas. Las crecidas y los caudales altos fueron responsables del $74 \%$ de la carga total de sedimentos en suspensión. Aguas abajo, la frecuencia de la carga sedimentaria se ve alterada por las presas de Mequinenza y Ribarroja. La variabilidad natural de los afluentes desaparece y la carga de sedimentos es más constante en el tiempo. La duración requerida para transportar el $86 \%$ de la carga en suspensión en la sección de SCR fue del $42 \%$ del tiempo $(Q>$ $187 \mathrm{~m}^{3} \mathrm{~s}^{-1}$ ). Este patrón está en el mismo rango de variabilidad que el observado en el sistema SegreCinca, sugiriendo por una parte que estos dos afluentes aún ejercen cierta influencia en la carga 
sedimentaria aguas abajo de Ribarroja, a pesar del alto grado de regulación de los dos sistemas (SegreCinca y Ebro). Las crecidas y los caudales altos son responsables del $38 \%$ de la carga total de sedimentos en suspensión transportada en SCR, un valor notablemente más bajo que el obtenido en SCSA y en los afluentes ibéricos.
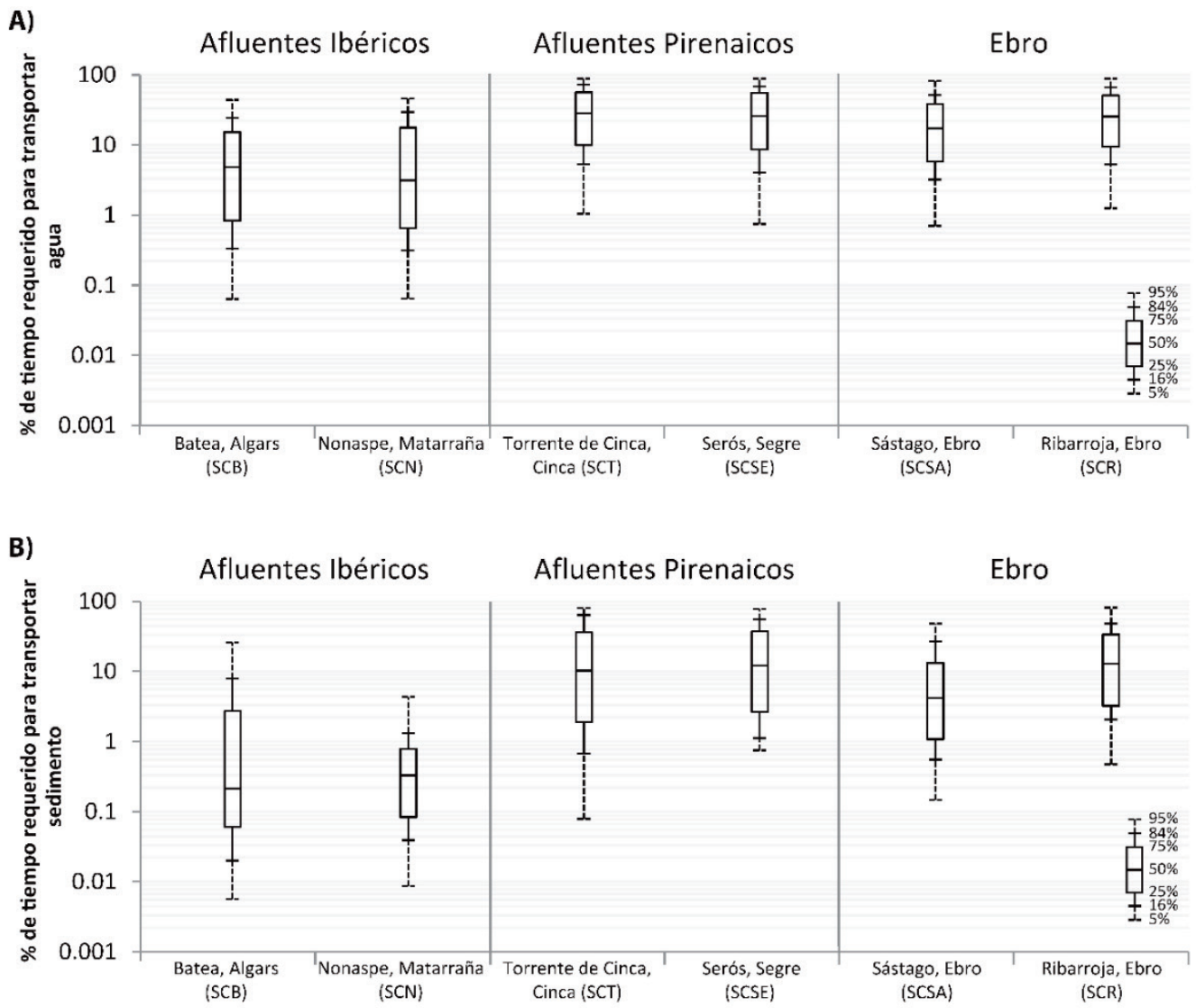

Figura 2. Boxplot (diagrama de caja y bigotes) que muestra el porcentaje de tiempo requerido para alcanzar diferentes percentiles de la aportación hídrica $(5,16,25,50,5,84$ y 95\%) y de la carga sedimentaria en las diferentes secciones de control durante el periodo de estudio (2008-2011). A) Representa la aportación hídrica en las diferentes secciones B) Representa la carga sedimentaria en las diferentes secciones. En la figura las diferentes cajas están separadas por su localización geográfica: Afluentes ibéricos: SCB (Sección de Control de Batea, Río Algars) y SCN (Sección de Control de Nonaspe, Río Matarraña); Afluentes pirenaicos: SCT (Sección de Control de Torrente, Río Cinca) y SCSE (Sección de Control de Seròs, Río Segre); Bajo Ebro aguas arriba del embalse de Mequinenza: SCSA (Sección de Control de Sástago) y aguas abajo de la presa de Ribarroja; SCR (Sección de Control de Ribarroja). Ver Figura 1B para la localización de las secciones.

\subsection{Balance sedimentario del embalse de Ribarroja}

La construcción del balance sedimentario en el embalse de Ribarroja tiene en cuenta la carga sólida estimada aguas arriba del embalse de Mequinenza, las principales entradas de sedimentos en el embalse de Ribarroja y la carga observada aguas abajo del mismo. El objetivo principal es la cuantificación de los flujos sedimentarios en el embalse (entradas y salidas), y el análisis de la influencia de: $i$ ) el diferente régimen hidrológico de las cuencas fluyentes $\mathrm{y}$, ii) la influencia del complejo de presas de Mequinenza y Ribarroja (CPMR) en el tránsito de sedimentos. La Figura 3 resume el balance sedimentario del embalse de Ribarroja y representa los principales flujos de sedimentos para el período de estudio. 


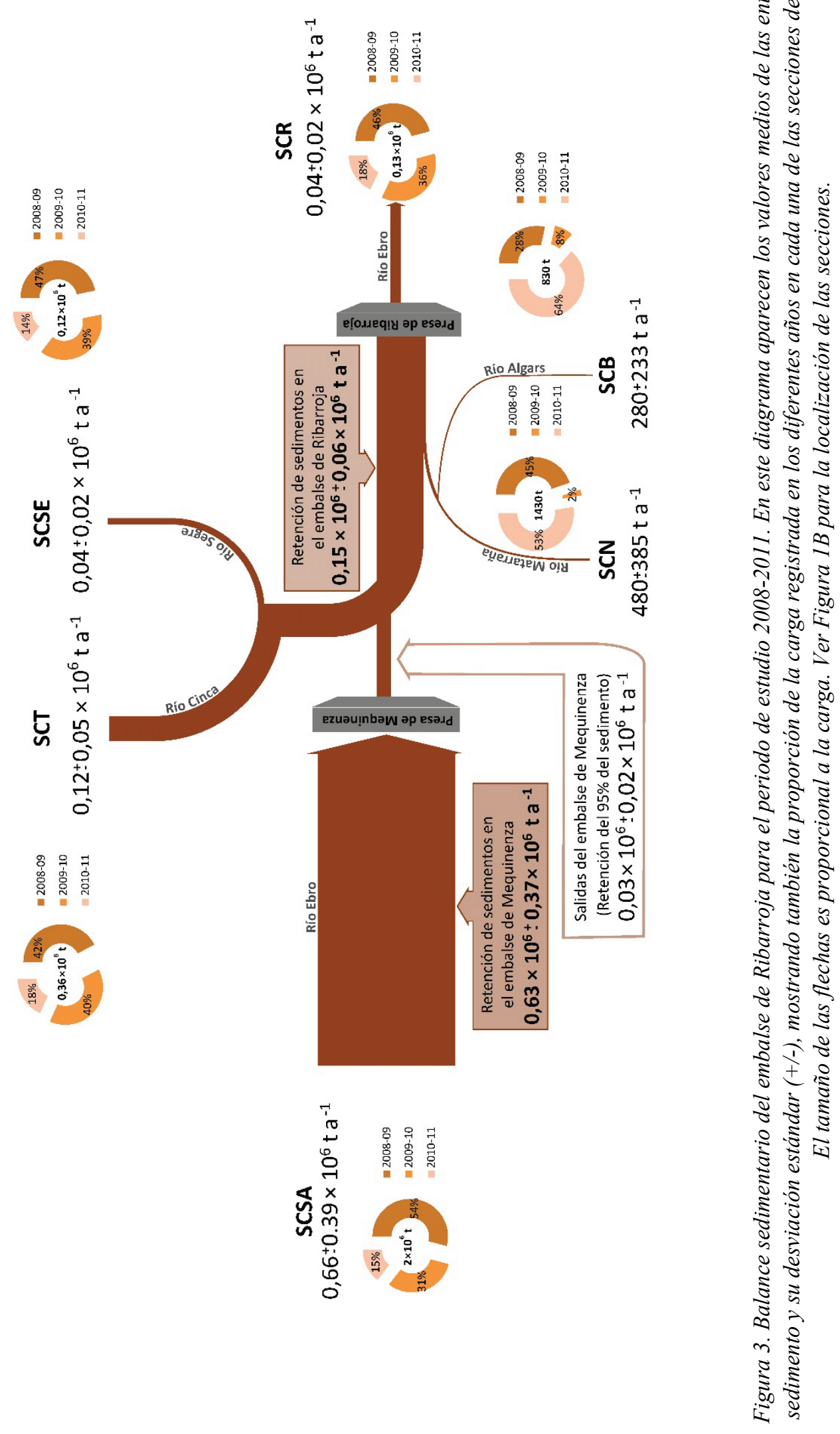




\subsubsection{Entrada de sedimentos al embalse de Mequinenza}

La aportación hídrica y los caudales máximos observados durante el período de estudio fueron similares a los años previamente estudiados por Vericat y Batalla (2006). Por lo tanto, las curvas de duración de caudales para el período 2008-2011, junto con la relación estadísticamente significativa entre $Q$ y $C S S$ obtenida en 2003-2004, proporcionan una base fiable para la estimación de la carga sedimentaria que ha transitado por SCSA durante el período de estudio. A modo de comparación, Vericat y Batalla (2006) calcularon una carga sedimentaria de $1 \times 10^{6} \mathrm{t} \mathrm{a}^{-1}$ para una aportación hídrica de $7263 \mathrm{hm}^{3}$; mientras que en el caso de la carga sedimentaria en 2008-2009 fue de 1,1 $\times 10^{6} \mathrm{t} \mathrm{a}^{-1}$ para una aportación hídrica de $7384 \mathrm{hm}^{3}$. Roura (2004) determinó una carga sedimentaria de $0,5 \times 10^{6}$ t para años con un promedio de aportación hídrica cercano a $5700 \mathrm{hm}^{3}$, un valor que coincide con las estimaciones de este estudio para los años más secos i.e. 2009-10, con una aportación hídrica de $6275 \mathrm{hm}^{3}$ y una carga de $0,6 \times 10^{6} \mathrm{t}$ de sedimento, y $2010-11$ con $4121 \mathrm{hm}^{3}$ de agua y $0,3 \times 10^{6} \mathrm{t} \mathrm{de}$ sedimento. La carga sedimentaria estimada para el río Ebro aguas arriba del embalse de Mequinenza fue de 2,0 $\times 10^{6} \mathrm{t}$ para todo el período 2008-2011, promediando una carga anual de $0,7 \times 10^{6} \mathrm{t} \mathrm{a}^{-1}(\sigma=0,4 \mathrm{t})$. Este valor representa una carga específica cercana a $13 \mathrm{t} \mathrm{km}^{-2} \mathrm{a}^{-1}$, un valor ligeramente inferior al obtenido por Sanz et al. (1999) y Vericat y Batalla (2006) durante períodos más húmedos $\left(30 \mathrm{t} \mathrm{km}^{-2} \mathrm{a}^{-1}\right)$, y superiores a los estimados por Palanques $\left(1987,7 \mathrm{t} \mathrm{km}^{-2} \mathrm{a}^{-1}\right)$ y Roura $\left(2004,10 \mathrm{t} \mathrm{km}^{-2} \mathrm{a}^{-1}\right)$ durante los años más secos. La carga estimada que entra en el embalse de Mequinenza es del orden de magnitud de otros grandes ríos europeos como el Garona y el Rin, cuya carga específica es de $21 \mathrm{t} \mathrm{km}^{-2} \mathrm{a}^{-1} \mathrm{y}$ $15 \mathrm{t} \mathrm{km}^{2} \mathrm{a}^{-1}$, respectivamente (Meybeck y Ragu, 1997). Aunque esta sección ya esté afectada por un déficit de sedimento estructural derivado de los embalses aguas arriba (ver Batalla y Vericat, 2011 para más detalles), aún mantiene un cierto comportamiento natural en cuanto a la dinámica del transporte sedimentario. Este hecho queda demostrado por la notable variabilidad interanual observada en la carga sedimentaria entre los años más húmedos y más secos (ca. 60\%). Debido a su tamaño y a la enorme capacidad de regulación, el tiempo de residencia del agua en el embalse de Mequinenza es bastante elevado (entre 1 y 2,5 meses según Prats et al. 2009), favoreciendo así la sedimentación de la mayor parte de la carga en suspensión transportada por el Ebro. Roura (2004) estima una capacidad de retención de sedimentos del 95\% para un período de estudio de 2 años (1998-2000).

\subsubsection{Entrada de sedimentos al embalse de Ribarroja}

La fracción de la carga de sedimentos en suspensión que pasa a través de la presa de Mequinenza entra directamente en el embalse de Ribarroja ya que la cola del mismo llega hasta la presa. Basado en la capacidad de retención de sedimentos del embalse de Mequinenza del 95\% obtenida por Roura (2004), nuestras estimaciones indican que llegaron al embalse de Ribarroja alrededor de $0,1 \times 10^{6}$ t durante el período 2008-2011, lo que significa una aportación anual de $0,03 \mathrm{t}(\sigma=0,02 \mathrm{t})$.

Tres kilómetros aguas abajo de la presa de Mequinenza, el Ebro recibe la aportación de los dos afluentes principales de la cuenca (Segre y Cinca, ver Fig. 1B para más detalles). La carga sedimentaria de estos dos ríos para todo el período fue de $0,49 \times 10^{6} \mathrm{t}\left(\sigma=0,02 \times 10^{6} \mathrm{t}\right)$, produciendo una carga media anual de $0,08 \mathrm{t} \mathrm{a}^{-1}$ (aproximadamente $1 / 4$ proveniente del Segre $\mathrm{y} 3 / 4$ del Cinca, a lo largo del período de estudio de 3 años). La distribución de las cargas anuales se encuentra en la tabla 2 y en la Figura 3 de manera porcentual. Específicamente, la carga en SCSE (Río Segre) para el período de estudio fue de $0,12 \times 10^{6} \mathrm{t}\left(\sigma=0,02 \times 10^{6} \mathrm{t}\right)$, produciendo una carga media anual de $0,04 \times 10^{6} \mathrm{t}$ (ver Tabla 2); a su vez, la carga obtenida en SCT (Río Cinca) fue de $0,36 \times 10^{6} \mathrm{t}\left(\sigma=0,05 \times 10^{6} \mathrm{t}\right)$, con una carga media anual de $0,12 \mathrm{t} \mathrm{a}^{-1}$. Como en el caso de la aportación hídrica, la variabilidad interanual de la carga sedimentaria es relativamente baja ( $\mathrm{CV}=39 \%$ y $50 \%$, respectivamente). La carga sedimentaria media anual fue de 3,2 $\mathrm{t} \mathrm{km}^{-2} \mathrm{a}^{-1}$ en el caso del Segre y de $12,7 \mathrm{t} \mathrm{km}^{-2} \mathrm{a}^{-1}$ en el Cinca. A pesar de que la carga es notable en estas cuencas, sobre todo en el caso del Cinca, los valores permanecen por debajo de los obtenidos en otros ríos que drenan áreas montañosas en todo el mundo (Dedkov y Moszherin, 1992) 
debido a la elevada regulación de ambas cuencas, y a otras presiones antrópicas (e.g. extracciones de áridos) que condicionan el gran déficit sedimentario al que están sujetas.

Aproximadamente $28 \mathrm{~km}$ aguas abajo de la entrada de los afluentes pirenaicos, el Matarraña (incluido su afluente principal, el Algars) desemboca en el embalse de Ribarroja. La carga sedimentaria proveniente de estos afluentes Ibéricos fue de 2260 t para todo el período de estudio, produciendo un valor medio anual de 376,15 t a ${ }^{-1}(\sigma=304 \mathrm{t})$ (ver la distribución de las cargas en la Tabla 2 y la Fig. 3). Debido a su mayor superficie, la carga obtenida en el Matarraña es mayor que la del Algars. La carga total que transitó por la sección de control Nonaspe (SCN, Río Matarraña) fue de 1426 t, produciendo una carga media anual de $475 \mathrm{t}(\sigma=385 \mathrm{t}$; ver Tabla 2). Este valor es superior a las $831 \mathrm{t}$ que pasaron por la sección de control de Batea (SCB, Río Algars) con una carga media anual de 280 ( $\sigma=233$ t; ver Tabla 2). En ambos casos, la carga sedimentaria está marcada por una fuerte variabilidad interanual, variando entre 35 t (2009-2010) y 751 t (2010-2011) en el caso de Matarraña, y entre 67 t (2009-2010) y 528 t (2010 -2011) en el Algars (ver Tabla 2). Estos valores representan CV de 123\% y 84\% para SCN y $\mathrm{SCB}$, respectivamente. La carga específica del Algars $\left(0,85 \mathrm{t} \mathrm{km}^{-2}\right.$ año-1) es más alta que la del Matarraña $\left(0,46 \mathrm{t} \mathrm{km}^{-2}\right.$ año $\left.{ }^{-1}\right)$, aunque en ambos casos los valores son muy bajos en comparación con otras cuencas mediterráneas similares (Inbar 1992; Batalla et al., 2005; Lobera et al., 2016; Piqué et al., 2017) y un orden de magnitud inferior al observado en las cuencas pirenaicas. Estas cargas no están directamente relacionadas con la aportación hídrica cuyo máximo se registró en 2008-09; en cambio, podrían estar relacionadas con la mayor crecida registrada, que tuvo lugar en el año hidrológico 201011 y que fue responsable del 61\% (322 t) y el 45\% (339t) del sedimento transportado en este año en el Algars y el Matarraña, respectivamente.

En conjunto, la carga sedimentaria que entró en el embalse de Ribarroja fue aproximadamente de $0,60 \times 10^{6} \mathrm{t}$ para todo el período de estudio, produciendo un valor medio anual de $0,2 \times 10^{6} \mathrm{ta}^{-1}(\sigma=$ $\left.0,09 \times 10^{6} \mathrm{t}\right)$. Anualmente, la carga de sedimentos en suspensión se distribuyó con $0,27 \times 10^{6} \mathrm{t}$ en $2008-$ $09,0,22 \times 10^{6} \mathrm{t}$ en $2009-10$, y casi $0,1 \times 10^{6} \mathrm{t}$ en $2010-11$. Aunque el tamaño $(35 \mathrm{~km}$ de largo y 220 $\mathrm{hm}^{3}$ de capacidad) del embalse de Ribarroja es inferior al del embalse de Mequinenza, igualmente gran parte de los sedimentos en suspensión que a él llegan quedan atrapados en él. Vericat y Batalla (2006) estimaron una tasa de retención de hasta el $90 \%$ en el embalse de Ribarroja sin tener en cuenta la aportación de los afluentes.

\subsubsection{Salidas del embalse de Ribarroja}

El efecto del complejo de embalses Mequinenza-Ribarroja sobre la carga de sedimentos del río Ebro es notable. La carga sedimentaria en SCR, inmediatamente aguas abajo de la presa, fue de $0,13 \times$ $10^{6} \mathrm{t}$ durante todo el período de estudio, con un valor medio anual de $0,04 \mathrm{t} \mathrm{a}^{-1}\left(\sigma=0,02 \times 10^{6} \mathrm{t}\right)$. Este valor representa ca. $25 \%$ de la carga total entrante en Ribarroja y ca. $5 \%$ si también tenemos en cuenta las entradas a Mequinenza. Porcentajes similares de retención (i.e. 95\%) fueron estimados por Avendaño et al. (1997) basados en datos de sedimentación en el propio embalse; Sanz et al. (1999) basados en un muestreo infrecuente; Vericat y Batalla (2005) siguiendo el método de Brune (1953) y datos de dos crecidas extraordinarias, y Vericat y Batalla (2006) basados en un muestreo frecuente.

Las cargas anuales se dieron en relación directa con la hidrología observada. De este modo, se registró una mayor carga sedimentaria en 2008-09 y menor en 2009-10 y 2010-11 (ver Tabla 2). La variabilidad del sedimento entre años fue moderada (CV 46\%). Aunque no tiene un sentido geomorfológico claro por la desconexión del punto de muestreo con la cuenca aguas arriba, se ofrece un valor de carga específica: se trata de un valor muy bajo, $0,7 \mathrm{t} \mathrm{km}^{-2} \mathrm{a}^{-1}$, como cabía esperar para un sistema fuertemente regulado. Este valor es ligeramente más bajo que el estimado previamente por los autores para un período de 10 años en dos secciones situadas aguas abajo del embalse, la primera en Pas de l'Ase $\left(1 \mathrm{t} \mathrm{km}^{-2} \mathrm{a}^{-1}\right)$ y la segunda en Xerta $\left(1,3 \mathrm{t} \mathrm{km}^{-2} \mathrm{a}^{-1}\right)$ (Tena et al., 2011, 2012). Las diferencias entre las estimaciones de este estudio y otros estudios recientes en el bajo Ebro (por ejemplo, Vericat y 
Batalla 2006; Négrel et al., 2007) pueden atribuirse al carácter seco del período de estudio 2008-2011, y a las diferencias que pueda haber entre las secciones donde se ha estimado la carga (e.g. confluencia de tributarios que aporten carga sedimentaria). Aun así, la comparación de estos valores con otros grandes ríos regulados en Europa como por el ejemplo el Rin $\left(15 \mathrm{t} \mathrm{km}^{-2} \mathrm{a}^{-1}\right.$, Meybeck y Ragu, 1997) y el Ródano (101 t km$~^{-2} \mathrm{a}^{-1}$, Serrat et al., 2001) indica la severidad del déficit sedimentario en el Ebro en su tramo final.

\section{Discusión}

El balance de sedimentos realizado corrobora que el complejo de embalses de Mequinenza y Ribarroja reduce notablemente la carga sedimentaria transportada por el río. Como diversos estudios anteriores han puesto de manifiesto, el déficit sedimentario que generan las presas del tramo bajo del río se suma al que el resto de grandes presas genera a lo largo de la cuenca, todo ello en un contexto de manifiesta reducción de la aportación sedimentaria de la cuenca desde la mitad del siglo pasado (ver los detalles del balance sedimentario completo de la cuenca del Ebro en Batalla y Vericat, 2011). Este fenómeno está asociado a varios factores, tales como la reducción de la escorrentía y, con ello, de la erosión y la carga sedimentaria debido sobre todo a la reforestación que ha tenido lugar después del abandono de tierras de cultivo durante la segunda mitad del siglo XX (e.g. Gallart y Llorens, 2004). López-Moreno et al. (2008) atribuyen esta reducción en la escorrentía a una reducción en la precipitación y acumulación de nieve, sumado a un aumento de la evapotranspiración. Además de estos factores primarios, el papel de los embalses ubicados en las regiones de cabecera es también importante, ya que interrumpen la continuidad entre las zonas de generación de sedimentos, las de transporte, y las de sedimentación. Un buen ejemplo de esto es el embalse de Barasona, que regula el sistema ÉseraIsábena. Lobera et al. (2016) estimaron una sedimentación cercana a las $300.000 \mathrm{t} \mathrm{a}^{-1}$ para el periodo 2011-2013, teniendo en cuenta tan solo el río Ésera. Sin embargo, si se incluyera el río Isábena en estos cálculos, la cifra aumentaría hasta las $550.000 \mathrm{t} \mathrm{a}^{-1}$ (según López-Tarazón y Batalla, 2014).

La carga total de sedimentos en suspensión atrapados en el complejo Mequinenza-Ribarroja durante el período de estudio se estima en $2,35 \times 10^{6} \mathrm{t}$ (lo que equivale al $95 \%$ del sedimento transportado por el río Ebro aguas arriba del embalse de Mequinenza y los principales afluentes), valor que supone una retención media anual de $0,78 \times 10^{6} \mathrm{t}$. Es importante mencionar que el periodo de estudio de este trabajo se clasifica como seco; por lo tanto, la carga de sedimentos fue probablemente más baja en comparación con los valores obtenidos a más largo plazo (Batalla y Vericat, 2011).

La retención de sedimentos en los embalses genera un déficit sedimentario en los tramos aguas abajo. Richards (1982) señaló que el efecto de la retención de sedimentos tiende a disminuir aguas abajo, y la carga sedimentaria puede llegar a recuperar unas condiciones similares a las de aguas arriba de la presa. Sin embargo, la distancia necesaria para la recuperación puede variar según la contribución de los afluentes y el tamaño de la cuenca, entre otros factores. Williams y Wolman (1984) establecieron que para grandes ríos se requería una distancia de $500 \mathrm{~km}$ para recuperar los niveles previos a la presa, aunque, en algunos casos, la carga de sedimentos pudiera no llegar a recuperarse completamente. En el caso del Ebro, el complejo de embalses analizado se encuentra a $115 \mathrm{~km}$ de la desembocadura en el mar, valor inferior al citado por dichos autores como referencia. Cabe citar que en dicho tramo los afluentes son efímeros, y el único río con capacidad suficiente para aportar cantidades apreciables de sedimentos, el Siurana, está también regulado y su lecho afectado históricamente por una importante minería de gravas. Trabajos anteriores apuntan a que, durante episodios de crecida, ya sean naturales o artificiales, por medio de la erosión de los márgenes y el lecho se puede aumentar la carga sedimentaria del río hasta algunos cientos de miles de toneladas por año (Batalla y Vericat, 2009; Vericat y Batalla, 2006; Tena et al., 2013). Otros trabajos han demostrado que las contribuciones de los pequeños afluentes efimeros durante eventos torrenciales pueden incluso superar la carga transportada durante una crecida de mantenimiento (5\% de la carga media anual para el período 1998-2008; Tena et al., 2012). 
El balance de sedimentos del embalse de Ribarroja proporciona información interesante para comprender el efecto de las presas en el comportamiento sedimentario de un gran río mediterráneo, todo ello en el contexto de un déficit estructural de sedimentos de la propia cuenca desarrollado a lo largo del siglo XX. El conocimiento adquirido en este trabajo puede y debe considerarse como una base para diseñar estrategias para la gestión de sedimentos, conducentes a la progresiva renaturalización (o rehabilitación parcial o total) de su régimen hidrológico y sedimentario. En el bajo Ebro se llevan a cabo medidas de rehabilitación hidrológica desde el año 2002 mediante desembalses regulares desde Ribarroja (crecidas de mantenimiento, Vericat y Batalla, 2009; Tena et al., 2014). Estas crecidas artificiales han sido diseñadas e implementadas, monitorizadas y modelizadas con el objetivo de controlar las poblaciones de macrófitos y analizar el transporte de sedimentos durante las mismas. Los resultados demostraron inicialmente su efectividad en la eliminación de macrófitos (hasta el 95\% en el meandro de Flix, aunque disminuyendo en dirección aguas abajo) y su potencial para erosionar y transportar sedimentos aguas abajo (Batalla y Vericat, 2009; Vericat y Batalla, 2006; Tena et al., 2013).

El potencial demostrado por estas medidas de rehabilitación, junto con la predisposición del organismo de cuenca y los gestores de las infraestructuras hidráulicas para aplicar estas medidas en otros embalses de la cuenca puede abrir nuevos horizontes y pasar de la concepción tradicional de las crecidas de mantenimiento desde una sola presa, hacia una crecida de mantenimiento en cascada en otros ríos regulados de la cuenca del Ebro. Se podrían coordinar de esta manera operaciones de mantenimiento de presas, como la suelta de sedimentos en el embalse de Barasona u otros, con conocidos problemas de sedimentación, junto con crecidas de mantenimiento generadas, por ejemplo, desde la presa de Grado en el río Cinca.

Finalmente, en un estudio dirigido por Dolz et al. (2009) sobre la dinámica sedimentaria del embalse de Ribarroja, se calculó un volumen de entre 13 y $16 \mathrm{hm}^{3}$ de sedimentos acumulados en todo el embalse (valores que corroboran los obtenidos en este estudio), de los cuales una importante proporción $\left(5 \mathrm{hm}^{3}\right)$ se encuentra en la confluencia del sistema Segre-Cinca con el Ebro. Además, estos mismos autores observaron que durante un solo evento de crecida gran parte de estos depósitos se movilizaron hasta 3 kilómetros. Esta información justificaría analizar el potencial de la coordinación de operaciones desde la cuenca del río Cinca y la presa de Ribarroja para maximizar la transferencia de sedimentos hacia el tramo bajo del río.

\section{Conclusiones}

El balance sedimentario del embalse de Ribarroja se ha elaborado a partir de datos de caudal y sedimento en suspensión en las entradas y salidas del mismo para el periodo (2008-2011). Las principales conclusiones del trabajo son:

1. La carga sedimentaria media anual entrante en el embalse de Ribarroja fue de $0,2 \times 10^{6} \mathrm{ta}^{-1}(\sigma=$ $0,09 \times 10^{6} \mathrm{t}$ ), mientras que el valor medio anual obtenido aguas abajo de la presa fue de $0,04 \times$ $10^{6} \mathrm{ta}^{-1}\left(\sigma=0,02 \times 10^{6} \mathrm{t}\right)$.

2. La tasa de retención del embalse de Ribarroja es de aproximadamente el $75 \%$ de la carga total entrante en el embalse; si se tienen en cuenta las entradas de sedimento al embalse de Mequinenza la tasa de retención aumenta hasta el 95\%.

3. Los resultados ponen de manifiesto el papel de los embalses, en este caso el de Ribarroja, en la retención de sedimentos a lo largo de la cuenca del Ebro y particularmente en su tramo bajo, hecho que exacerba el déficit sedimentario del río como consecuencia de la progresiva disminución en el suministro de sedimentos que se ha producido en la cuenca lo largo del siglo XX. 


\section{Agradecimientos}

Este trabajo se ha desarrollado con datos obtenidos en el marco de los proyectos CGL200611679-C02-01/HID y CGL2009-09770 (subprograma BTE) financiados por el Ministerio de Educación y Ciencia. El segundo autor está contratado como profesor agregado mediante el programa Serra Húnter de la Generalitat de Catalunya. El Grupo de Investigación de Dinámica Fluvial-RIUS agradece el apoyo de la Generalitat de Catalunya a través del grupo de investigación (2017 SGR 0459) y el programa CERCA. Los datos hidrológicos han sido suministrados por la Confederación Hidrográfica del Ebro.

\section{Referencias}

Avendaño, C., Cobo, R., Sanz, M.E., Gómez, J.L. 1997. Capacity situation in Spanish reservoirs. I.C.O.L.D. Ninenteenth Congress on Large Dams 74 (52), 849-862.

Balasch, J.C., Pino, D., Ruiz-Bellet, J.L., Tuset, J., Barriendos, M., Castelltort, X., Peña, J.C. 2019. The extreme floods in the Ebro River basin since 1600 CE. Science of the Total Environment 646, 645-660. https://doi.org/10.1016/j.scitotenv.2018.07.325

Batalla, R.J., Kondolf, G.M., Gomez, C.M. 2004. Reservoir-induced hydrological changes in the Ebro River basin, NE Spain. Journal of Hydrology 290, 117-136. https://doi.org/10.1016/j.jhydrol.2003.12.002

Batalla, R.J., Garcia, C., Balasch, J.C. 2005. Total sediment load in a Mediterranean mountainous catchment (the Ribera Salada River, Catalan Pre-Pyrenees, NE Spain). Zeitschrift für Geomorphology 49(4), 495-514.

Batalla, R.J., Vericat, D. 2009. Hydrological and sediment transport dynamics of flushing flows: implications for river management in large Mediterranean rivers. River Research and Applications 25(3), 297-314. https://doi.org/10.1002/rra.1160

Batalla, R.J., Vericat, D. 2011. An appraisal of the contemporary sediment yield in the Ebro Basin. Journal of Soils and Sediments 11, 1070-1081. https://doi.org/10.1007/s11368-011-0378-8

Brune, G.M. 1953. The trap efficiency of reservoirs. Transactions of the American Geophysical Union 34, 407418. https://doi.org/10.1029/TR034i003p00407

Dedkov, A.P., Mozzherin, V.I. 1992. Erosion and sediment yield on the Earth. In Erosion and Sediment Yield: Global and Regional Perspectives. IAHS Publications 236, 29-33.

Dolz, J., Armengol, J., Roura, M., De Pourcq, K., Arbat, M., López, P. 2009. Estudio de la dinámica sedimentaria y batimetría de precisión del embalse de Ribarroja. Barcelona.

Frutos, L.M., Ollero, A., Sánchez, M. 2004. Caracterización del río Ebro y su cuenca y variaciones en su comportamiento hidrológico. En Gil Olcina, A. (Coord.): Alteración de los regímenes fluviales peninsulares, Fundación Caja Murcia, Murcia, pp. 233-280.

Gallart, F., Llorens, P. 2004. Observations on land cover changes and water resources in the headwaters of the Ebro catchment, Iberian Peninsula. Physics and Chemistry of the Earth 769-773 parts A/B/C (11-12). https://doi.org/10.1016/j.pce.2004.05.004

Hu, B., Yang, Z., Wang, H., Sun, X., Bi, N. 2009. Sedimentation in the Three Gorges Dam and its impact on the sediment flux from the Changjiang (Yangtze River), China. Hydrology and Earth System Sciences Discussion 6, 5177-204. https://doi.org/10.5194/hessd-6-5177-2009

Inbar, M. 1992. Rates of fluvial erosion in basins with a Mediterranean climate type. Catena 19, 393-409. https://doi.org/10.1016/0341-8162(92)90011-Y

Lobera, G., Batalla, R.J., Vericat, D., López-Tarazón, J.A., Tena, A. 2016. Sediment transport in two Mediterranean regulated rivers. Science of the Total Environment 540, 101-113. https://doi.org/10.1016/J.SCITOTENV.2015.08.018

López-Moreno, J.I., García-Ruiz, J.M., Beniston, M. 2008. Environmental Change and water management in the Pyrenees. Facts and future perspectives for Mediterranean mountains. Global and Planetary Change 66 (3-4), 300-312. https://doi.org/10.1016/j.gloplacha.2007.10.004 
López-Tarazón, J.A., Batalla, R.J. 2014. Dominant discharges for suspended sediment transport in a highly active Pyrenean river. Journal of Soils and Sediments 14(12), 2019-2030. https://doi.org/10.1007/s11368-0140961-x

Meybeck, M., Ragu, A. 1997. River discharges to the oceans: an assessment of suspended solids, major ions and nutrients. UN Environment Programme, Nairobi, Kenya. 245 p.

Milliman, J.D., Syvitski, J.P.M. 1992. Geomorphic/tectonic control of sediment discharge to the ocean: the importance of small mountainous rivers. The Journal of Geology 100, 525-544. https://doi.org/10.1086/629606

Milliman, J.D., Farnsworth, K.L. 2011. River Discharge to the Coastal Ocean: A Global Synthesis. Cambridge University Press, Cambridge, 143-144. https://doi.org/10.1017/cbo9780511781247

Négrel, P., Roy, S., Petelet-Giraud, E., Millot, R., Brenot, A. 2007. Long-term fluxes of dissolved and suspended matter in the Ebro River Basin (Spain). Journal of Hydrology 342, 249-260. https://doi.org/10.1016/j.jhydrol.2007.05.013

Novoa, M. 1984. Precipitaciones y avenidas extraordinarias en Catalunya. Actas de las Jornadas de Trabajo sobre Inestabilidad de laderas en el Pirineo. Barcelona, 1-15.

Palanques, A. 1987. Dinámica sedimentaria, meneralogía y microcontaminantes inorgánicos de las suspensiones $y$ de los sedimentos superficiales en el margen continental del Ebro. Tesis Doctoral, Universidad de Barcelona.

Piqué, G., Batalla, R.J., López, R., Sabater, S. 2017. The fluvial sediment budget of a dammed river (upper Muga, southern Pyrenees). Geomorphology 293, 211-226. http://doi.org/10.1016/j.geomorph.2017.05.018

Poulos, S.E., Collins, M.B. 2002. A quantitative evaluation of riverine water/sediment fluxes to the Mediterranean basin: natural flows, coastal zone evolution and the role the dam construction. In: Jones SJ, Frostick LE (eds.) Sediment Flux to Basins: Causes, Controls and Consequences. Geological Society, London, Special Publications 191, pp. 227-245.

Prats, J., Dolz, J., Armengol, J. 2009. Variabilidad temporal en el comportamiento hidráulico del curso inferior del río Ebro. Ingeniería del Agua, 16(4), 259- 272. https://doi.org/10.4995/ia.2009.2960

Richards, K.S. 1982. Rivers: Form and Process in Alluvial Channels. Methuen, London, 358 p.

Roura, M. 2004. Incidència de l'embassament de Mequinensa en el transport de sòlids en suspensió i la qualitat de l'aigua del riu Ebre. Tesis doctoral. Universidad de Barcelona.

Rovira, A., Ibáñez, C. 2007. Sediment linkages between the river catchment and the sea. Journal of Soils and Sediments, 7 (5), 285-295. https://doi.org/10.1065/jss2007.08.244

Rovira, A., Ibáñez, C., Martín-Vide, J.P. 2015. Suspended sediment load at the lowermost Ebro River (Catalonia, Spain). Quaternary International 388, 188-198. https://doi.org/10.1016/j.quaint.2015.05.035

Sanz, M.E., Avendaño, C., Cobo, R. 1999. Influencia de los embalses en el transporte de sedimentos hasta el río Ebro (España). Proceedings of the Congress on Hydrological and geochemical processes in large-scale river basins. HIBAM, Shahin.

Serrat, P., Ludwig, W., Navarro, B., Blazi, J.L. 2001. Variabilité spatio-temporelle des flux de matières en suspension d'un fleuve côtier méditerranéen : la Têt (France)/ Spatial and temporal variability of sediment fluxes from a coastal Mediterranean river: the Têt (France). Comptes Rendus de l'Académie des Sciences (Series II A). Earth and Planetary Science 333, 389-397. https://doi.org/10.1016/S1251-8050(01)01652-4

Shaw, E.M. 1983. Hydrology in Practice. Van Nostrand Reinhold, London.

Syvitski, J.P.M., Kettner, A. 2011. Sediment Flux and the Anthropocene. Philosophical Transactions of the Royal Society A: Mathematical, Physical and Engineering Sciences 369, 957-975. https://doi.org/10.1098/rsta.2010.0329

Tena, A., Batalla, R.J., Vericat, D., Lopez-Tarazón, J.A. 2011. Suspended sediment dynamics in a large regulated river over a 10-year period (the lower Ebro, NE Iberian Peninsula). Geomorphology 125, 73-84. https://doi.org/10.1016/j.geomorph.2010.07.029 
Tena, A., Batalla, R.J., Vericat, D. 2012. Reach-scale suspended sediment balance downstream from dams in a large Mediterranean river. Hydrological Sciences Jounal 57, 1-19. https://doi.org/10.1080/02626667.2012.681784

Tena, A., Książek, L., Batalla, R.J., Vericat, D. 2013. Assessing the geomorphic effects of a flushing flow in a large regulated river. River Research and Applications 29 (7), 876-890. https://doi.org/10.1002/rra.2572

Tena, A., Batalla, R. J. 2013. The sediment budget of a large river regulated by dams (The lower River Ebro, NE Spain). Journal of Soils and Sediments 13 (5), 966-980. https://doi.org/10.1007/s11368-013-0681-7

Tena, A., Vericat, D., Batalla, R.J. 2014. Suspended sediment dynamics during flushing flows in a large impounded river (the lower River Ebro). Journal of Soils and Sediments 14 (12), 2057-2069. https://doi.org/10.1007/s11368-014-0987-0

Topping, D.J., Rubin, D.M. and Vierra, L.E. 2000. Colorado River sediment transport: 1. Natural sediment supply limitation and the influence of Glen Canyon Dam. Water Resources Research 36. https://doi.org/10.1029/1999WR900285.

Vericat, D., Batalla, R.J. 2005. Sediment transport in a highly regulated fluvial system during two consecutive floods (Lower Ebro River, NE Spain). Earth Surface Processes and Landforms 30, 255-272.

Vericat, D., Batalla, R.J. 2006. Sediment transport in a large impounded river: the lower Ebro River, NE Iberian Peninsula. Geomorphology 79, 72-92. https://doi.org/10.1016/j.geomorph.2005.09.017

Vericat, D., Batalla, R.J., Garcia, C. 2006. Breakup and reestablishment of the armour layer in a highly regulated large gravel-bed river: the lower Ebro. Geomorphology 76 (1-2), 122-136. https://doi.org/10.1016/j.geomorph.2005.10.005.

Vörösmarty, C. J., Meybeck, M., Fekete, B., Sharma, K., Green, P., Syvitski, J.P.M. 2003. Anthropogenic sediment retention: major global impact from registered river impoundments. Global and Planetary Change 39 (12), 169-190. https://doi.org/10.1016/S0921-8181(03)00023-7

Walling, D.E. 2006. Human impact on land-ocean sediment transfer by the world's rivers. Geomorphology 79 , 192-216.

Walling, D.E., Fang, D. 2003. Recent trends in the suspended sediment loads of the world's rivers. Global and Planearyt Change 39, 111-126. https://doi.org/10.1016/S0921-8181(03)00020-1

Williams, G.P., Wolman, M.G. 1984. Downstream effects of dams on alluvial rivers. United States Geological Survey Professional Paper 1286, 83 pp. 